\title{
Intermixed medullary and papillary thyroid cancer in a patient with renal cell carcinoma
}

\author{
Shanika Samarasinghe ${ }^{1}$, Simge Yuksel ${ }^{2}$ and Swati Mehrotra ${ }^{3}$ \\ 1Division of Endocrinology and Metabolism, 2Division of Internal Medicine, and ${ }^{3}$ Department of Pathology and \\ Laboratory Medicine, Loyola University Medical Center, Maywood, Illinois, USA
}

Correspondence should be addressed to S Samarasinghe Email

ssamarasinghe@lumc.edu

\section{Summary}

We report a rare case of concurrent medullary thyroid cancer (MTC) and papillary thyroid cancer (PTC) with intermixed disease in several of the lymph node (LN) metastases in a patient who was subsequently diagnosed with clear cell renal cell carcinoma (RCC). A 56 year old female presented with dysphagia and was found to have a left thyroid nodule and left superior cervical LN with suspicious sonographic features. Fine needle aspiration biopsy (FNAB) demonstrated PTC in the left thyroid nodule and MTC in the left cervical LN. Histopathology demonstrated multifocal PTC with 3/21 LNs positive for metastatic PTC. One LN in the left lateral neck dissection exhibited features of both MTC and PTC within the same node. In the right lobe, a $0.3 \mathrm{~cm}$ focus of MTC with extra-thyroidal extension was noted. Given persistent calcitonin elevation, a follow-up ultrasound displayed an abnormal left level 4 LN. FNAB showed features of both PTC and MTC on the cytopathology itself. The patient underwent repeat central and left radical neck dissection with 3/6 LNs positive for PTC in the central neck and 2/6 LNs positive for intermixed PTC and MTC in the left neck. There was no evidence of distant metastases on computed tomography and whole body scintigraphy, however a $1.9 \times 2.5 \mathrm{~cm}$ enhancing mass within the right inter-polar kidney was discovered. This lesion was highly suspicious for RCC. Surgical pathology revealed a $2.5 \mathrm{~cm}$ clear cell RCC, Fuhrman grade 2/4, with negative surgical margins. She continues to be observed with stable imaging of her triple malignancies.

\section{Learning points:}

- Mixed medullary-papillary thyroid neoplasm is characterized by the presence of morphological and immunohistochemical features of both medullary and papillary thyroid cancers within the same lesion. Simultaneous occurrence of these carcinomas has been previously reported, but a mixed disease within the same lymph node is an infrequent phenomenon.

- Prognosis of mixed medullary-papillary thyroid carcinomas is determined by the medullary component. Therefore, when PTC and MTC occur concurrently, the priority should be given to the management of MTC, which involves total thyroidectomy and central lymph node dissection.

- Patients with thyroid cancer, predominantly PTC, have shown higher than expected rates of RCC. To our knowledge, this is the first report describing the combination of MTC, PTC, and RCC in a single patient. 


\section{Background}

It is rare for three distinct malignancies to be described in the same patient. Medullary thyroid cancer (MTC) is a relatively uncommon thyroid cancer accounting for $3-5 \%$ of all thyroid cancers compared to papillary thyroid cancer (PTC), which represents approximately $85 \%$ of all thyroid malignancies (1). The origin of MTC is embryologically distinct from that of PTC. Calcitonin producing parafollicular $\mathrm{C}$ cells derive from ectodermal neural crest, whereas follicular cells are of endodermal origin. As such, the incidence, histopathology, and prognosis vary significantly between these tumors.

The simultaneous occurrence of MTC and PTC occurs with reported prevalence of $0.28-2.6 \%$ (2). Several case reports and studies describe synchronous occurrence of these two carcinomas, but a mixed lesion within the same lymph tissue is an infrequent event. We report a case of concurrent medullary and papillary carcinoma of the thyroid gland with mixed disease in several of the lymph node metastases. A unique feature of this presentation is that the patient was also subsequently diagnosed with clear cell renal cell carcinoma (RCC). The literature supports an association between PTC and primary RCC (3), but to our knowledge, this is the first report describing the combination of MTC, PTC and RCC in a single patient.

\section{Case presentation}

A 56-year-old female with a history of type 2 diabetes mellitus, non-alcoholic steatohepatitis, and hypertension presented with dysphagia and submandibular pain. The patient had no apparent family history of endocrine disorders, although her mother had a history of breast cancer and a brother had RCC. A left thyroid nodule was appreciated on the patient's exam and ultrasonography demonstrated a left $2.5 \times 1.4 \times 1.8-\mathrm{cm}$ ) hypoechoic nodule with micro-calcifications and a left superior $2.0 \times 1.4 \times 1.8$ $\mathrm{cm}$ lobulated cervical lymph node. Her serum thyroid stimulating hormone (TSH) and calcium levels were normal. Her thyroglobulin antibodies (anti-Tg) and antithyroperoxidase antibodies were negative.

\section{Investigation}

Given the suspicious features on ultrasonography, fineneedle aspiration biopsy (FNAB) was undertaken for both lesions. The DiffQuik and Papanicolaou stained cytology smears from the left lobe of the thyroid showed complex papillary architecture, flat sheets of cells with oval nuclei, powdery chromatin, nuclear grooves, nuclear membrane irregularities, and rare intranuclear inclusions. A diagnosis of PTC was rendered (Fig. 1A and B). Cytologic smears from the left cervical lymph node were morphologically different and exhibited a discohesive population of plasmacytoid cells with nuclear pleomorphism and salt and pepper chromatin (Fig. 1C and D). An immunostain for calcitonin was positive, confirming the cytologic impression of metastatic MTC. CT imaging of the neck and chest confirmed a hypodense $2.1 \times 1.5 \times 1.5$-cm left thyroid mass without evidence of extension beyond the left lobe as well as several highly suspicious left neck lymph nodes, the largest measuring $1.0 \times 0.8 \times 1.5 \mathrm{~cm}$. A $7-\mathrm{mm}$ soft tissue nodule was also noted in the right paratracheal region. No focal lung lesions were seen. The patient's pre-operative calcitonin and carcinoembryonic antigen (CEA) levels were $251 \mathrm{pg} / \mathrm{mL}$ (normal range: 0-5.1 $\mathrm{pg} / \mathrm{mL}$ ) and $4.7 \mathrm{ng} / \mathrm{mL}$ (normal range: $0-2.5 \mathrm{ng} / \mathrm{mL}$ ), respectively. Plasma metanephrines were normal. She subsequently underwent a total thyroidectomy with bilateral modified lateral and central neck dissections.

\section{Treatment}

The surgical specimen demonstrated classic variant bilateral multifocal PTC, the largest foci in the left lobe

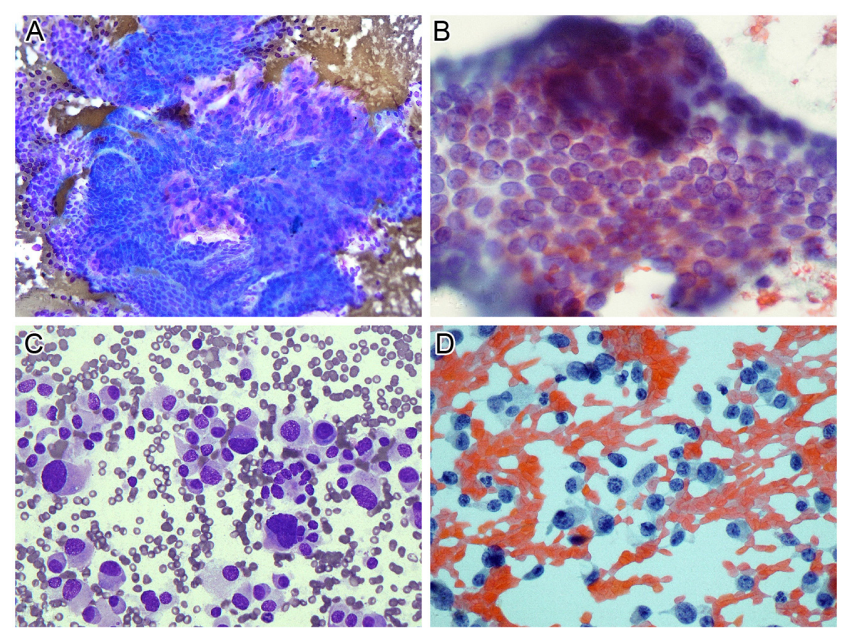

\section{Figure 1}

(A) DiffQuik stained smear from the left lobe of the thyroid shows complex arborizing papillary architecture. (B) Papanicolaou stained smear from the left lobe of thyroid highlights the partially folded sheet of follicular cells with oval nuclei, powdery nuclear chromatin, nuclear membrane irregularity, and nuclear grooves - features diagnostic of papillary carcinoma. (C) DiffQuik stained smear from left cervical lymph node shows discohesive plasmacytoid cells with obvious nuclear pleomorphism. (D) Papanicolaou stained smear from the left cervical lymph node highlights the salt and pepper chromatin - features diagnostic of medullary carcinoma. 
measuring $1.6 \mathrm{~cm}$ with $3 / 21$ regional lymph nodes positive for metastatic PTC. One lymph node in the left lateral neck dissection exhibited features of both MTC and PTC within the same node. MTC formed the bulk of the nodal tumor. In the right lobe, a $0.3-\mathrm{cm}$ focus of MTC with extra-thyroidal extension was noted with no lymph nodes involved by metastatic carcinoma in the right lateral neck. Extensive c-cell hyperplasia was identified on multiple slides within both lobes of the thyroid gland. No mixed primary tumor was discovered within the thyroid gland. Immunohistochemical stains for calcitonin, thyroglobulin ( $\mathrm{Tg}$ ), and synaptophysin were used to confirm the diagnosis. Tumor staging according to American Joint Committee on Cancer (AJCC) seventh edition (in use at the time of surgery) was T3, N1b, and Mx for the MTC and pT1b, N1b, and Mx for the PTC. The patient's calcitonin and CEA levels improved to $48 \mathrm{pg} /$ $\mathrm{mL}$ and $2.3 \mathrm{ng} / \mathrm{mL}$ post-operatively. She received 148.8 $\mathrm{mCi}$ of I-131 for management of the PTC. Stimulated Tg measured $31.4 \mathrm{ng} / \mathrm{mL}$ with anti-Tg $<1 \mathrm{IU} / \mathrm{mL}$ (normal range $0-4 \mathrm{IU} / \mathrm{mL}$ ) prior to radioiodine ablation. Post therapy whole body scintigraphy demonstrated thyroid bed uptake at $2.3 \%$ at $72 \mathrm{~h}$ with no evidence of distant metastases. RET proto-oncogene mutational analysis was negative.

\section{Outcome and follow-up}

As sustained calcitonin and Tg elevation raised concern for persistent disease, a follow-up ultrasound 6 months later displayed an abnormal 1.4-cm left level 4 lymph node. FNAB showed features of both PTC and MTC on the cytopathology itself (Fig. 2A). Immunohistochemical stains on the aspiration displayed PTC, while a subset of the tumor cells also stained positive for calcitonin. Eight months after her original surgery, the patient underwent repeat central and left radical neck dissection. Histopathology demonstrated 3/6 lymph nodes positive for PTC in the central level 6 neck dissection and $2 / 6$ lymph nodes positive for intermixed papillary and medullary carcinoma in the left level 4 dissection. The excised lymph nodes displayed the complex arborizing papillary structures of PTC intimately admixed with solid islands composed of the small ovoid spindle cells of MTC (Fig. 2B, C and D). Calcitonin decreased further from 59 $\mathrm{pg} / \mathrm{mL}$ pre-operatively to $13 \mathrm{pg} / \mathrm{mL}$ following resection and suppressed $\mathrm{Tg}$ decreased from $15.2 \mathrm{ng} / \mathrm{mL}$ to $1.6 \mathrm{ng} /$ $\mathrm{mL}$. Her post-operative course was complicated by an occipital lobe posterior cerebral artery stroke with visual field defects from which the patient eventually recovered.

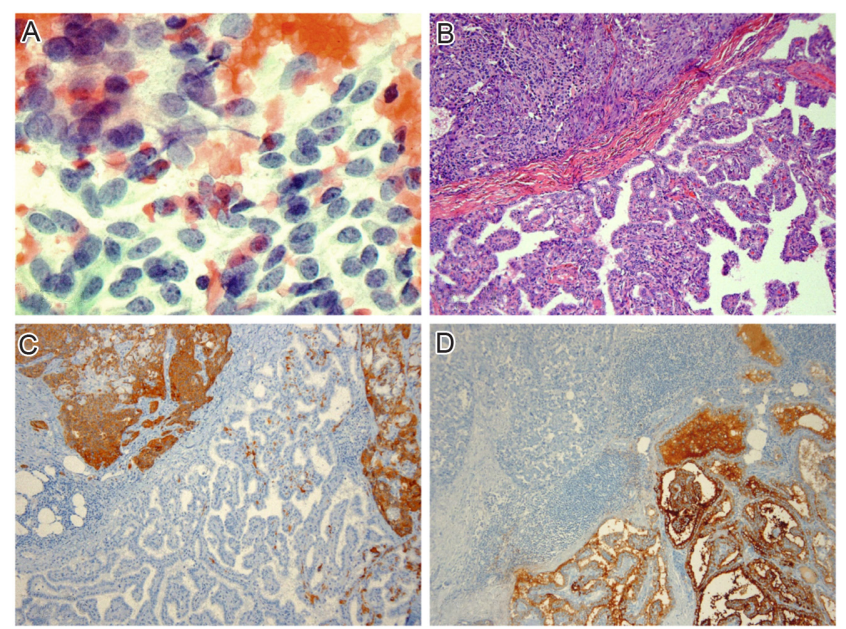

\section{Figure 2}

(A) Papanicolaou stained smear highlighting mixed papillary and medullary carcinoma metastatic to the same lymph node. The nuclear features of papillary carcinoma are appreciated in the tumor cluster at the upper left corner. The plump spindle cells of medullary carcinoma display salt and pepper chromatin. (B) Hematoxylin and eosin stained section of the excised lymph node shows solid islands of medullary carcinoma at the upper left and papillary architecture of papillary carcinoma on the lower right. (C) Calcitonin immunostain highlights the medullary carcinoma; papillary carcinoma is not immunoreactive. (D) Thyroglobulin immunostain highlights the papillary carcinoma; islands of medullary carcinoma are not immunoreactive.

She continued to undergo surveillance with CT and whole body scintigraphy with a stimulated $\mathrm{Tg}$ of $5.5 \mathrm{ng} /$ $\mathrm{mL}$ on early follow-up, but there was no evidence of distant metastases; however, a $1.9 \times 2.5$-cm enhancing mass within the right inter-polar kidney was discovered. This lesion was highly suspicious for RCC. Two years after her original thyroidectomy, the patient underwent a right robotic-assisted laparoscopic partial nephrectomy with histopathology revealing a 2.5 -cm clear cell RCC, Fuhrman grade $2 / 4$, with negative surgical margins. Further genetic evaluation was advised but deferred by the patient due to cost considerations. The patient has been observed for 9 years of follow-up with stable CT imaging of her multiple malignancies. Biochemical markers have shown a mild increase in calcitonin at $33 \mathrm{pg} / \mathrm{mL}$, suppressed $\mathrm{Tg}$ at 2.8 $\mathrm{ng} / \mathrm{mL}$, and a normal CEA at $1.9 \mathrm{ng} / \mathrm{mL}$, but with no overt structural correlate on imaging. She continues to undergo close surveillance.

\section{Discussion}

The incidence of PTC has increased over the last decades, primarily due to advances in diagnostics, and is associated with thyroglobulin production. In contrast, MTC is infrequent and shows immunoreactivity for CEA, 
calcitonin, and other neuroendocrine markers such as chromogranin and synaptophysin. The prognosis of MTC is generally worse than PTC. The concurrent occurrence of MTC and PTC can be observed in the form of a collision or mixed tumor. Collision tumors present as anatomically distinct PTC and MTC separated by normal thyroid tissue. In mixed tumors, PTC and MTC are intermingled within the same lesion and demonstrate morphological and immunohistochemical properties of both components. We present a rare case of simultaneous PTC and MTC with mixed tumor within several of the lymph node metastases. FNAB of these lymph nodes demonstrated both PTC and MTC features on the cytopathology itself. Immunohistochemical studies on the same aspiration displayed reactivity to calcitonin, thyroglobulin, and synaptophysin characterizing the distinct tumor types within the same lesion. Despite extensive sampling, a mixed primary tumor within the thyroid gland was not detected. This unusual phenomenon of two discrete tumors metastasizing to several but not all of the same regional lymph nodes supports a theory of independent events.

A recent Italian multicenter study investigated the epidemiologic characteristics and clinical outcomes in 183 patients with simultaneous PTC-MTC (4). Overall, $45 \%$ of patients were disease-free after $>10$ years from diagnosis, with $72.5 \%$ for PTC and $51.1 \%$ for MTC (4). These results highlight that the prognosis of synchronous PTC-MTC is often driven by the medullary component and that priority should be given to the management of MTC which involves total thyroidectomy and central lymph node dissection (4). Measurement of calcitonin in the pre-surgical work-up of thyroid nodules with positive cytology for PTC has also been advocated to aid in early diagnosis of concomitant MTC.

Thyroid carcinoma is associated with various genetic mutations which result in differing disease behavior. Somatic RET/PTC rearrangements and mutations in $R A S$ and $B R A F$ oncogenes have been identified in PTC and can be used in tumor prognostication (1). RET activating point mutations have been documented in MTC. Multiple theories have been proposed to explain the pathogenesis of mixed medullary-papillary carcinoma. Most reports support the 'collision' phenomenon suggesting that two independent tumors located in the same lesion are coincidental events. It has been theorized that intermixed tumors could derive from a common uncommitted stem cell or potentially a common tumorigenic stimuli triggers neoplastic transformation of both follicular and C cells (5). Volante et al. argued against the single stem cell hypothesis by completing a mutational analysis demonstrating distinct patterns of RET proto-oncogene mutation, loss of heterozygosity, and X- chromosomal inactivation (clonality) in the two histological components of mixed tumors (5). In another genetic study of three cases of MTC-PTC collision tumor, RET mutations were identified in the medullary carcinoma component and a $B R A F$ mutation was identified in the papillary carcinoma component (6). These findings support distinct clonal origins in coexisting PTC/MTC.

The literature supports an increased incidence of thyroid cancer, with malignancy originating in other organs such as breast and kidney. Studies of second primary malignancies among patients with thyroid cancer, predominantly PTC, have shown higher than expected rates of RCC (3). Although a case of coexisting MTC, PTC, and urothelial carcinoma of the renal pelvis has been reported, to our knowledge this is the first study describing combination of MTC, PTC, and RCC (7). The established risk factors for kidney cancer such as obesity, smoking, and hypertension are not classically associated with thyroid cancer which include ionizing radiation and genetic factors. Several studies have also proposed possible genetic linkages between thyroid cancer and renal cancer. Malchoff et al. defined a distinct familial tumor syndrome characterized as the familial association of PTC, nodular thyroid disease, and papillary renal neoplasia (PRN). The familial PTC/PRN phenotype was linked to a germline mutation in chromosome 1q21 (8). Thyroid cancer of follicular origin has also been found with greater frequency in Cowden syndrome, an autosomal dominant disorder associated with a germline mutation in PTEN, a tumor suppressor gene located on chromosome 10q22-23 (9). It is characterized by multiple hamartomas and carcinomas of the breast, thyroid, kidney, colon, and uterus. Mutations in the CHEK2 gene, which plays an important role in DNA repair, are also associated with an increased risk of breast, colon, kidney, and thyroid cancer (10). Further research is required to delineate common risk factors and molecular mechanisms in thyroid cancer and RCC.

In summary, we report a unique case of a patient with synchronous PTC-MTC with intermixed disease in the lymph node metastases who was subsequently diagnosed with clear cell RCC. Treatment of mixed thyroid cancer is driven by the medullary component which requires total thyroidectomy and central lymph node dissection. While the association between PTC and primary RCC is a recognized phenomenon, to our knowledge, this study is the first evidence of coexistence of MTC, PTC, and RCC. It 
is crucial to recognize simultaneous primary malignancies for appropriate clinical management, as awareness can lead to earlier diagnosis and improved patient outcomes.

\section{Declaration of interest}

The authors declare that there is no conflict of interest that could be perceived as prejudicing the impartiality of this case report.

\section{Funding}

This research did not receive any specific grant from any funding agency in the public, commercial, or not-for-profit sector.

\section{Patient consent}

Written informed consent has been obtained from the patient for publication of the case report and accompanying images.

\section{Author contribution statement}

All co-authors contributed to writing and editing of this manuscript and approved the final version for submission for publication. S S was involved in the clinical care of the patient, composed the case study, and edited the manuscript. S M was one of the pathologists involved in the patient's case and provided the histopathology composites and descriptors. S Y conducted the literature review and drafted the discussion section. The authors thank the patient for giving permission to publish this case.

\section{References}

1 Fagin JA \& Wells Jr SA. Biologic and clinical perspectives on thyroid cancer. New England Journal of Medicine 2016375 1054-1067. (https://doi.org/10.1056/NEJMra1501993)

2 Erhamamci S, Reyhan M, Kocer NE, Nursal GN, Torun N \& Yapar AF. Simultaneous occurrence of medullary and differentiated thyroid carcinomas. Report of 4 cases and brief review of the literature. Hellenic Journal of Nuclear Medicine 201417 148-152. (https://doi. org/10.1967/s002449910137)

3 Carhill AA, Litofsky DR \& Sherman SI. Unique characteristics and outcomes of patients diagnosed with both primary thyroid and primary renal cell carcinoma. Endocrine Practice 201521 461-467. (https://doi.org/10.4158/EP14411.OR)

4 Appetecchia M, Lauretta R, Barnabei A, Pieruzzi L, Terrenato I, Cavedon E, Mian C, Castagna MG, Elisei R \& SIE (Italian Society of Endocrinology) Working Group. Epidemiology of simultaneous medullary and papillary thyroid carcinomas (MTC/PTC): an Italian multicenter study. Cancers 201911 1516. (https://doi.org/10.3390/ cancers11101516)

5 Volante M, Papotti M, Roth J, Saremaslani P, Speel EJ, Lloyd RV, Carney JA, Heitz PU, Bussolati G \& Komminoth P. Mixed medullaryfollicular thyroid carcinoma. Molecular evidence for a dual origin of tumor components. American Journal of Pathology 1999155 1499-1509. (https://doi.org/10.1016/S0002-9440(10)65465-X)

6 Rossi S, Fugazzola L, De Pasquale L, Braidotti P, Cirello V, BeckPeccoz P, Bosari S \& Bastagli A. Medullary and papillary carcinoma of the thyroid gland occurring as a collision tumour: report of three cases with molecular analysis and review of the literature. Endocrine-Related Cancer 200512 281-289. (https://doi.org/10.1677/ erc.1.00901)

7 Albores-Saavedra J, Dorantes-Heredia R, Chablé-Montero F, CórdovaRamón JC \& Henson DE. Association of urothelial carcinoma of the renal pelvis with papillary and medullary thyroid carcinomas. A new sporadic neoplastic syndrome? Annals of Diagnostic Pathology 201418 286-290. (https://doi.org/10.1016/j.anndiagpath.2014.08.003)

8 Malchoff CD, Sarfarazi M, Tendler B, Forouhar F, Whalen G, Joshi V Arnold A \& Malchoff DM. Papillary thyroid carcinoma associated with papillary renal neoplasia: genetic linkage analysis of a distinct heritable tumor syndrome. Journal of Clinical Endocrinology and Metabolism 200085 1758-1764. (https://doi.org/10.1210/ jcem.85.5.6557)

9 Guilmette J \& Nosé V. Hereditary and familial thyroid tumours. Histopathology 201872 70-81. (https://doi.org/10.1111/his.13373)

10 Cybulsi C, Gorski B, Huzarski T, Masojc B, Mierzejewski M, Debniak T, Teodorczyk U, Byrski T, Gronwald J, Matyjasik J et al. CHEK2 is a multiorgan cancer susceptibility gene. American Journal of Human Genetics 200475 1131-1135. (https://doi. org/10.1086/426403)

Received in final form 8 April 2020

Accepted 30 April 2020 